\title{
Group testing with geometry of classical groups over finite fields
}

\author{
Suogang Gao' ${ }^{1}$ Zengti $\mathrm{Li}^{2} \cdot$ Weili $\mathrm{Wu}^{3}$. \\ Panos M. Pardalos ${ }^{4}$. Dingzhu $\mathrm{Du}^{3}$
}

\begin{abstract}
In this paper, we give an overview of combinatorial group testing and algebra. Our survey focuses on the constructions with algebraic methods, especially geometry of classical groups over finite fields.
\end{abstract}

Keywords Group testing · Pooling design · Classical groups

Mathematics Subject Classification 05E30 $\cdot 05$ E35 $\cdot 33$ C45 $\cdot$ 33D45

\section{Introduction}

The basic problem of DNA library screening is to determine which clone (a DNA segment) from the library contains which probe from a given collection of probes in an efficient fashion. A clone is said to be positive for a probe if it contains the probe, and negative otherwise.

This problem is just an instance of the general group testing problem, in which a large population of items containing a small set of defectives are to be tested to identify the defectives efficiently.

Suppose there are $n$ clones including at most $d$ positive ones (others are negative). A (group) test is applicable to an arbitrary subset of clones with two possible outcomes: a

Suogang Gao

sggaomail@163.com

1 Mathematics and Information College, Hebei Normal University, Shijiazhuang 050024, China

2 Mathematics and Information College, Langfang Normal University, Langfang 065000, China

3 Department of Computer Science, University of Texas at Dallas, Richardson, TX 75080, USA

4 Industrial and Systems Engineering, University of Florida, Gainesville, FL 32611, USA 
negative outcome indicates all clones in the subset are negative, and a positive outcome indicates otherwise. A pooling design is a specification of all tests so that they can be performed simultaneously with the goal being to identify all positive clones with a small number of tests. A pooling design $M$ can be represented by a binary incidence matrix where the columns represent clones, the rows represent tests, and $m_{i j}=1$ if and only if clone $j$ is contained in the subset of test $i$.

Suppose $M$ has $t$ rows. Then the $t$ outcomes can also be represented by a $t$-vector $V=\left(v_{1}, \ldots, v_{t}\right)^{T}$, where $v_{i}=1$ if and only if the outcome of test $i$ is positive $\left(v_{i}=0\right.$ otherwise). Note that $V$ is the Boolean sum of the set of positive clones. Therefore, it is convenient to view a column vector $C$ as a subset $S$ of the base set $\{1,2, \ldots, t\}$, where $i \in S$ if and only if $C$ has an 1 entry in row $i$. Then, we can say that $V$ is the union of the set of positive clones. $M$ is called $d$-disjunct if no union of any $d$ columns covers another column. A $d$-disjunct matrix not only identifies the up-to- $d$ positive clones, but it does so with a simple decoding. Namely, a clone is positive if and only if it (as a column) is contained by $V$. This is because a negative clone (column) has at least one row not covered by the union of the up-to- $d$ positive clones; such a row then has a negative outcome which identifies the clone as negative.

The notion of $d$-disjunctness was first raised by Kautz and Singleton [14] in the study of superimposed codes. It was also studied by Erdös et al. [3] under the name of $d$-cover-free family in extremal set theory. The $d$-disjunct matrices have become the most important tool in the construction of deterministic pooling designs. Although many constructions have been proposed, the existence of $d$-disjunct matrices is still sparse.

Macula [21] proposed a novel way of constructing $d$-disjunct matrices which uses the containment relation in a structure. More specifically, let $[m]:=\{1,2, \ldots, m\}$ be the base set. Then each of the $n$ columns is labeled by a (distinct) $k$ subset of $[m]$, assuming $n \leq\left(\begin{array}{c}m \\ k\end{array}\right)$, and each of the $\left(\begin{array}{c}m \\ d\end{array}\right)$ rows is labeled by a (distinct) $d$-subset of $[m]$, where $d<k<m ; m_{i j}=1$ if and only if the label of row $i$ is contained in the label of column $j$. He proved that $M$ is $d$-disjunct.

Huang and Weng [12] generalized the construction to an arbitrary atomic semilattice where the elements can be ranked. Again, label the columns by a subset of the rank $k$ elements, and label the rows by all rank $d$ elements, $d<k$, and then $M$ is $d$-disjunct.

Ngo and Du [27] further extended the construction to some geometric structures, such as simplicial complexes, and some graph properties, such as matchings. It is safe to say the 'containment matrix' method has opened a new door for constructing $d$-disjunct matrices from many mathematical structures. However, the basic result in all these constructions is invariably that, to obtain a $d$-disjunct matrix, use all rank $d$ elements for rows.

One practical problem with this type of construction is that a large $n$ forces $d$ to be large. Then the number of tests could be too large as there are too many rank $d$ elements. This led Macula [23] to propose using the rank 2 elements for rows, regardless of the real $d$. He showed that while there is no guarantee all positive clones will be identified, the probability of success is still satisfactory when $d$ does not deviate too much from 2. Ngo and Du made a similar comment.

D'Yachkov et al. [2] showed that the containment matrix which uses rank $r$ of elements for rows has the degree $d$ of disjunctness, where $r$ can be much less than $d$. 
In fact, $r$ can be any number from 1 to $k-1$ ( $k$ is the lever for columns), while $d \leq q^{r}$ for some constant $q$. This is the first happy surprise.

Note that geometric lattices are among pooling spaces. Huang et al. [13] attempted to draw possible connections from finite geometry and distance-regular graphs to pooling spaces: including the projective spaces, the affine spaces, the attenuated spaces, and a few families of geometric lattices associated with the orbits of subspaces under finite classical groups, and associated with $d$-bounded distance-regular graphs.

Guo et al. [7] introduced the concept of pooling semilattices and proved that a pooling semilattice is a pooling space and then showed how to construct pooling designs from a pooling semilattice. Moreover, they gave many examples of pooling semilattices and thus obtained the corresponding pooling designs.

Guo and Wang [5] gave a new module of pooling design. More specifically, for positive integers $k \leq n$, let $\left(\begin{array}{c}{[n]} \\ k\end{array}\right)$ be the set of all $k$-subsets of $[n]$. Given integers $1 \leq d<k<n$ and $0 \leq i \leq d$. Let $M(i ; d, k, n)$ be the binary matrix with rows indexed with $\left(\begin{array}{c}{[n]} \\ d\end{array}\right)$ and columns indexed with $\left(\begin{array}{c}{[n]} \\ k\end{array}\right)$ such that $M(A, B)=1$ if and only if $|A \cap B|=i$. It is the first module that is not a containment relation. For more information on pooling designs see the monograph by Du and Hwang [1].

In this paper, we give an overview of constructions on pooling design with geometry of classical groups over finite fields.

The rest of the paper is organized as follows. Section 2 introduces geometry of classical groups over finite fields. Section 3 gives the constructions of pooling designs based on geometry of classical groups over finite fields.

\section{Preliminary}

In this section, we recall the geometry of classical groups over finite fields.

Let $\mathbb{F}_{q}$ be a finite field with $q$ elements, where $q$ is a prime power, $\mathbb{F}_{q}^{(n)}$ be the $n$-dimensional row vector space over $\mathbb{F}_{q}$, and $G L_{n}\left(\mathbb{F}_{q}\right)$ be the general linear group of degree $n$ over $\mathbb{F}_{q} . G L_{n}\left(\mathbb{F}_{q}\right)$ acts on $\mathbb{F}_{q}^{(n)}$ in the following way:

$$
\begin{aligned}
& \mathbb{F}_{q}^{(n)} \times G L_{n}\left(\mathbb{F}_{q}\right) \rightarrow \mathbb{F}_{q}^{(n)}, \\
& \left(\left(x_{1}, x_{2}, \cdots, x_{n}\right), T\right) \mapsto\left(x_{1}, x_{2}, \cdots, x_{n}\right) T
\end{aligned}
$$

Let $P$ be an $m$-dimensional subspace of $\mathbb{F}_{q}^{(n)}$ and $v_{1}, v_{2}, \cdots, v_{m}$ be a basis of $P$, then

$$
\left(\begin{array}{c}
v_{1} \\
v_{2} \\
\vdots \\
v_{m}
\end{array}\right)
$$

is an $m \times n$ matrix over $\mathbb{F}_{q}$ of rank $m$. We call the matrix (2) a matrix representation of the subspace $P$ and use also the same letter $P$ to denote the matrix (2) if no ambiguity 
arises. The action (1) of $G L_{n}\left(\mathbb{F}_{q}\right)$ on $\mathbb{F}_{q}^{(n)}$ induces an action on the set of subspaces of $\mathbb{F}_{q}^{(n)}$ such that $T \in G L_{n}\left(\mathbb{F}_{q}\right)$ carries the subspace $P$ into $P T$.

Use any one of the other classical groups, such as the singular general linear group $G L_{n+l, n}\left(\mathbb{F}_{q}\right)$, the symplectic group $S_{p_{n}}\left(\mathbb{F}_{q}\right)$ (where $n=2 v$ ), the pseudo-symplectic group $P s_{2 v+\delta}\left(\mathbb{F}_{q}\right)$, the unitary group $U_{n}\left(\mathbb{F}_{q}\right)$ (where $q$ is a square), the orthogonal group $O_{n}\left(\mathbb{F}_{q}\right.$ ) (where $n=2 v+\delta$ and $\delta=0,1$, or 2) and affine-symplectic group $\operatorname{ASp}_{2 v}\left(\mathbb{F}_{q}\right)$ to replace $G L_{n}\left(\mathbb{F}_{q}\right)$. Then we can obtain the corresponding geometry.

Now let us introduce the definition of the other classical groups and their corresponding geometries.

- For two nonnegative integers $n$ and $l, \mathbb{F}_{q}^{(n+l)}$ denotes the $(n+l)$-dimensional row vector space over $\mathbb{F}_{q}$. The set of all $(n+l) \times(n+l)$ nonsingular matrices over $\mathbb{F}_{q}$ of the form

$$
\left(\begin{array}{cc}
T_{11} & T_{12} \\
0 & T_{22}
\end{array}\right)
$$

where $T_{11}$ and $T_{22}$ are nonsingular $n \times n$ and $l \times l$ matrices, respectively, forms a group under matrix multiplication, called the singular general linear group of degree $n+l$ over $\mathbb{F}_{q}$ and denoted by $G L_{n+l, n}\left(\mathbb{F}_{q}\right)$. If $l=0($ resp. $n=0), G L_{n, n}\left(\mathbb{F}_{q}\right)=G L_{n}\left(\mathbb{F}_{q}\right)$ (resp. $G L_{l, 0}\left(\mathbb{F}_{q}\right)=G L_{l}\left(\mathbb{F}_{q}\right)$ ) is the general linear group of degree $n$ (resp. $l$ ).

The vector space $\mathbb{F}_{q}^{(n+l)}$ together with the general linear group action is called the $(n+l)$-dimensional singular linear space over $\mathbb{F}_{q}$. For $1 \leq i \leq n+l$, let $e_{i}$ be the row vector in $\mathbb{F}_{q}^{(n+l)}$ whose $i$-th coordinate is 1 and all other coordinates are 0 . Denote by $E$ the $l$-dimensional subspace of $\mathbb{F}_{q}^{(n+l)}$ generated by $e_{n+1}, e_{n+2}, \ldots, e_{n+l}$. An $m$ dimensional subspace $P$ of $\mathbb{F}_{q}^{(n+l)}$ is called a subspace of type $(m, k)$ if $\operatorname{dim}(P \cap E)=k$. The collection of all the subspaces of types $(m, 0)$ in $\mathbb{F}_{q}^{(n+l)}$, where $0 \leq m \leq n$, is the attenuated space.

For a fixed subspace $P$ of type $(m, k)$ in $\mathbb{F}_{q}^{(n+l)}$, let $\mathcal{M}\left(m_{1}, k_{1} ; m, k ; n+l, n\right)$ denote the set of all the subspaces of type $\left(m_{1}, k_{1}\right)$ contained in $P$, and let $N\left(m_{1}, k_{1} ; m, k ; n+\right.$ $l, n)=\left|\mathcal{M}\left(m_{1}, k_{1} ; m, k ; n+l, n\right)\right|$. Then from [31],

$$
N\left(m_{1}, k_{1} ; m, k ; n+l, n\right)=q^{\left(m_{1}-k_{1}\right)\left(k-k_{1}\right)}\left[\begin{array}{c}
m-k \\
m_{1}-k_{1}
\end{array}\right]_{q}\left[\begin{array}{c}
k \\
k_{1}
\end{array}\right]_{q}
$$

- Let $n=2 v$. It is well known that the cogredience normal form of $2 v \times 2 v$ nonsingular alternate matrices is

$$
K=\left(\begin{array}{cc}
0 & I^{(v)} \\
-I^{(v)} & 0
\end{array}\right)
$$

Let

$$
S_{p_{2 v}}\left(\mathbb{F}_{q}\right)=\left\{T \in G L_{2 v}\left(\mathbb{F}_{q}\right) \mid T K T^{\mathrm{T}}=K\right\} .
$$

Then $S_{p_{2 v}}\left(\mathbb{F}_{q}\right)$ is a group with respect to the matrix multiplication, called the symplectic group of degree $2 v$ over $\mathbb{F}_{q}$. 
The vector space $\mathbb{F}_{q}^{(2 v)}$ together with the symplectic group action is called the $2 v$-dimensional space over $\mathbb{F}_{q}$. Let $P$ be an $m$-dimensional subspace of $\mathbb{F}_{q}^{(2 v)}$. An $m$-dimensional subspace $P$ is said to be of type $(m, r)$, if $P K P^{\mathrm{T}}$ is of rank $2 r$. In particular, subspaces of type $(m, 0)$ are called $m$-dimensional totally isotropic subspaces. The subspaces of type $(m, r)$ exist if and only if $2 r \leq m \leq v+r$. The subspace of type $(m, r)$, which contains subspaces of type $\left(m_{1}, r\right)$, exists if and only if $2 r \leq m_{1} \leq m \leq v+r$.

Let $N\left(m_{1}, r ; m, r ; 2 v\right)$ denote the number of subspaces of type $\left(m_{1}, r\right)$ contained in a given subspace of type $(m, r)$. It is known that

$$
N\left(m_{1}, r ; m, r ; 2 v\right)=q^{2 r\left(m-m_{1}\right)} \frac{\prod_{i=m-m_{1}+1}^{m-2 r}\left(q^{i}-1\right)}{\prod_{i=1}^{m_{1}-2 r}\left(q^{i}-1\right)} .
$$

- Let $\mathbb{F}_{q}$ be a finite field of characteristic 2 , and let

$$
S_{1}=\left(\begin{array}{ccc}
0 & I^{(v)} & \\
I^{(v)} & 0 & \\
& & 1
\end{array}\right), S_{2}=\left(\begin{array}{cccc}
0 & I^{(v)} & \\
I^{(v)} & 0 & & \\
& & 0 & 1 \\
& & 1 & 1
\end{array}\right)
$$

The pseudo-symplectic group of degree $2 v+\delta(\delta=1$, or 2$)$ over $\mathbb{F}_{q}$, denoted by $P s_{2 v+\delta}\left(\mathbb{F}_{q}\right)$, consists of all $(2 v+\delta) \times(2 v+\delta)$ matrix $T$ over $\mathbb{F}_{q}$ satisfying $T S_{\delta} T^{\mathrm{T}}=S_{\delta}$.

The vector space $\mathbb{F}_{q}^{(2 v+\delta)}$ together with the pseudo-symplectic group action is called the $(2 v+\delta)$-dimensional pseudo-symplectic space over $\mathbb{F}_{q}$ of characteristic 2.

Let $P$ be an $m$-dimensional subspace of $\mathbb{F}_{q}^{(2 v+\delta)} \cdot P S_{\delta} P^{\mathrm{T}}$ is cogredient to one of the following three forms

$$
\begin{aligned}
M(m, 2 r, r) & =\left(\begin{array}{lll}
0 & I^{(r)} & \\
I^{(r)} & 0 & \\
& & 0^{(m-2 r)}
\end{array}\right), \\
M(m, 2 r+1, r) & =\left(\begin{array}{llll}
0 & I^{(r)} & \\
I^{(r)} & 0 & & \\
& & 1 & \\
& & & 0^{(m-2 r-1)}
\end{array}\right),
\end{aligned}
$$

and

$$
M(m, 2 r+2, r)=\left(\begin{array}{llllll}
0 & I^{(r)} & & & \\
I^{(r)} & 0 & & & & \\
& & 0 & 1 & \\
& & 1 & 1 & \\
& & & & 0^{(m-2 r-2)}
\end{array}\right)
$$

for some $r$ such that $0 \leq r \leq\left\lfloor\frac{m}{2}\right\rfloor$. We say that $P$ is a subspace of type $(m, 2 r+\tau, r, \varepsilon)$, where $\tau=0,1$, or 2 and $\varepsilon=0$, or 1 , if 
(i) $P S_{\delta} P^{\mathrm{T}}$ is cogredient to $M(m, 2 r+\tau, r)$, and

(ii) $e_{2 v+1} \notin P$ or $e_{2 v+1} \in P$ according to $\varepsilon=0$ or 1 , respectively.

In particular, subspaces of type $(m, 0,0,0)$ and $(m, 0,0,1)$ are called $m$ dimensional totally isotropic subspaces. The subspaces of type $(m, 2 r+1, r, 1)$ exist if and only if $2 r+1 \leq m \leq v+r+1$. The subspaces of type $(m, 2 r+1, r, 1)$, which contain subspaces of type $\left(m_{1}, 2 r+1, r, 1\right)$, exist if and only if $2 r+1 \leq m_{1}<m \leq$ $v+r+1$.

Let $N\left(m_{1}, 0,0,0 ; m, 0,0,0 ; 2 v+\delta\right)$ denote the number of subspaces of type $\left(m_{1}, 0,0,0\right)$ contained in a given subspace of type $(m, 0,0,0)$. From [30],

$$
N\left(m_{1}, 0,0,0 ; m, 0,0,0 ; 2 v+\delta\right)=\frac{\prod_{i=m-m_{1}+1}^{m}\left(q^{i}-1\right)}{\prod_{i=1}^{m_{1}}\left(q^{i}-1\right)} .
$$

- Let $q=q_{0}^{2}$, where $q_{0}$ is a prime power. $\mathbb{F}_{q}=\mathbb{F}_{q_{0}^{2}}$ has an involutive automorphism

$$
-: a \rightarrow \bar{a},
$$

whose fixed field is $\mathbb{F}_{q_{0}}$. Let

$$
U_{n}\left(\mathbb{F}_{q}\right)=\left\{T \in G L_{n}\left(\mathbb{F}_{q}\right) \mid T \bar{T}^{\mathrm{T}}=I^{(n)}\right\} .
$$

Then $U_{n}\left(\mathbb{F}_{q}\right)$ is a group with respect to the matrix multiplication, called the unitary group of degree $n$ over $\mathbb{F}_{q}$.

The vector space $\mathbb{F}_{q}^{(n)}$ together with the unitary group action is called the $n$ dimensional unitary space over $\mathbb{F}_{q}$. An $m$-dimensional subspace $P$ is said to be of type $(m, r)$, if $P H_{\delta}(\bar{P})^{\mathrm{T}}$ is of rank $r$. In particular, subspaces of type $(m, 0)$ are called $m$-dimensional totally isotropic subspaces. The subspaces of type $(m, r)$ exist if and only if $2 r \leq 2 m \leq n+r$. The subspace of type $(m, r)$, which contains subspaces of type $\left(m_{1}, r_{1}\right)$, exists if and only if $2 r \leq 2 m \leq n+r, 2 r_{1} \leq 2 m_{1} \leq n+r_{1}$ and $0 \leq r-r_{1} \leq 2\left(m-m_{1}\right)$. Let $N\left(m_{1}, r ; m, r ; n\right)$ denote the number of subspaces of type $\left(m_{1}, r\right)$ contained in a given subspace of type $(m, r)$. From [30]

$$
N\left(m_{1}, r ; m, r ; n\right)=q^{2 r\left(m-m_{1}\right)} \frac{\prod_{i=m-m_{1}+1}^{m-r}\left(q^{2 i}-1\right)}{\prod_{i=1}^{m_{1}-r}\left(q^{2 i}-1\right)} .
$$

- Let $\mathbb{F}_{q}$ be a finite field of odd characteristic. For a fixed non-square element $z$ of $\mathbb{F}_{q}^{*}$, let

$$
S_{2 r+\delta, \Delta}=\left(\begin{array}{ccc}
0 & I^{(v)} & \\
I^{(v)} & 0 & \\
& & \Delta
\end{array}\right), \text { where } \Delta= \begin{cases}\emptyset, & \text { if } \delta=0 \\
(1) \text { or }(z), & \text { if } \delta=1 \\
\operatorname{diag}(1,-z), & \text { if } \delta=2 .\end{cases}
$$

The orthogonal group of degree $2 v+\delta$ over $\mathbb{F}_{q}$, denoted by $O_{2 v+\delta, \Delta}\left(\mathbb{F}_{q}\right)$, consists of all $(2 v+\delta) \times(2 v+\delta)$ matrix $T$ over $\mathbb{F}_{q}$ satisfying $T S_{2 v+\delta, \Delta} T^{\mathrm{T}}=S_{2 v+\delta, \Delta}$. 
The vector space $\mathbb{F}_{q}^{(2 v+\delta)}$ together with the orthogonal group action is called the $(2 v+\delta)$-dimensional orthogonal space over $\mathbb{F}_{q}$ of odd characteristic.

Let $P$ be an $m$-dimensional subspace of $\mathbb{F}_{q}^{(2 v+\delta)} \cdot P S_{2 v+\delta} P^{\mathrm{T}}$ is cogredient to one of the following four forms

$$
\begin{aligned}
& M(m, 2 r, r)=\left(\begin{array}{lll}
0 & I^{(r)} & \\
I^{(r)} & 0 & \\
& & 0^{(m-2 r)}
\end{array}\right), \\
& M(m, 2 r+1, r, 1)=\left(\begin{array}{llll}
0 & I^{(r)} & & \\
I^{(r)} & 0 & & \\
& & 1 & \\
& & & 0^{(m-2 r-1)}
\end{array}\right), \\
& M(m, 2 r+1, r, z)=\left(\begin{array}{llll}
0 & I^{(r)} & & \\
I^{(r)} & 0 & & \\
& & z & \\
& & & 0^{(m-2 r-1)}
\end{array}\right), \\
& M(m, 2 r+2, r)=\left(\begin{array}{lllll}
0 & I^{(r)} & & & \\
I^{(r)} & 0 & & & \\
& & 1 & & \\
& & & -z & \\
& & & & 0^{(m-2 r-2)}
\end{array}\right) \text {. }
\end{aligned}
$$

We say that $P$ is a subspace of type $(m, 2 r+\gamma, r, \Gamma)$, if $P S_{2 v+\delta} P^{\mathrm{T}}$ is cogredient to $M(m, 2 r+\gamma, r, \Gamma)$, where $\Gamma=\emptyset$, if $\gamma=0$, and $\Gamma=0$ or $(z)$, if $\gamma=1$, and $\Gamma=\left(\begin{array}{cc}1 & \\ & -z\end{array}\right)$, if $\gamma=2$. In particular, subspaces of type $(m, 0,0)$ are called $m$ dimensional totally isotropic subspaces. The subspaces of type $(m, 2 r+1, r, 1)$ exist if and only if $2 r+1 \leq m \leq v+r+1$. The subspace of type $(m, 2 r+1, r, 1)$, which contains subspaces of type $\left(m_{1}, 2 r+1, r, 1\right)$, exists if and only if $2 r+1 \leq m_{1}<$ $m \leq v+r+1$. Let $N\left(m_{1}, 0,0 ; m, 0,0 ; 2 v+\delta, \Delta\right)$ denote the number of subspaces of type $\left(m_{1}, 0,0\right)$ contained in a given subspace of type $(m, 0,0)$. From [30],

$$
N\left(m_{1}, 0,0 ; m, 0,0 ; 2 v+\delta, \Delta\right)=\frac{\prod_{i=m-m_{1}+1}^{m}\left(q^{i}-1\right)}{\prod_{i=1}^{m_{1}}\left(q^{i}-1\right)} .
$$

- Let $\mathbb{F}_{q}$ be a finite field of characteristic 2. Denote by $\mathcal{K}_{n}$ the set of all $n \times n$ alternate matrices over $F_{q}$. Two $n \times n$ matrices $A$ and $B$ over $F_{q}$ are said to be congruent $\bmod \mathcal{K}_{n}$, denoted $A \equiv B\left(\bmod \mathcal{K}_{n}\right)$, if $A-B \in \mathcal{K}_{n}$. Clearly, $\equiv$ is an equivalence relation on the set of all $n \times n$ matrices. Let $[A]$ denote the equivalence class containing $A$. Two matrix classes $[A]$ and $[B]$ are said to be cogredient if there is a nonsingular $n \times n$ matrix $Q$ over $F_{q}$ such that $\left[Q A Q^{\mathrm{T}}\right] \equiv[B]$. 
Let

$$
G_{2 v+\delta, \Delta}=\left(\begin{array}{ccc}
0 & I^{(v)} & \\
& 0 & \\
& &
\end{array}\right), \text { where } \Delta= \begin{cases}\emptyset, & \text { if } \delta=0, \\
(1), & \text { if } \delta=1, \\
\left(\begin{array}{ll}
\alpha & 1 \\
& \alpha
\end{array}\right), & \text { if } \delta=2,\end{cases}
$$

where $\alpha$ is a fixed element of $F_{q}$ such that $\alpha \notin\left\{x^{2}+x \mid x \in F_{q}\right\}$.

The orthogonal group of degree $2 v+\delta$ over $F_{q}$ with respect to $G_{2 v+\delta, \Delta}$, denoted by $O_{2 v+\delta, \Delta}\left(F_{q}\right)$, consists of all $(2 v+\delta) \times(2 v+\delta)$ matrices $T$ over $F_{q}$ satisfying $\left[T G_{2 v+\delta, \Delta} T^{\mathrm{T}}\right] \equiv\left[G_{2 v+\delta, \Delta}\right]$.

The vector space $\mathbb{F}_{q}^{(2 v+\delta)}$ together with the orthogonal group action is called the $(2 v+\delta)$-dimensional orthogonal space over $\mathbb{F}_{q}$ of characteristic 2.

Let $P$ be an $m$-dimensional subspace of $\mathbb{F}_{q}^{(2 v+\delta)} . P G_{2 v+\delta} P^{\mathrm{T}}$ is cogredient to one of the following three forms

$$
\begin{aligned}
& M(m, 2 r, r)=\left(\begin{array}{ccc}
0 & I^{(r)} & \\
& 0 & \\
& & 0^{(m-2 r)}
\end{array}\right), \\
& M(m, 2 r+1, r)=\left(\begin{array}{cccc}
0 & I^{(r)} & & \\
& 0 & & \\
& & 0^{(m-2 r-1)} & \\
& & & 1
\end{array}\right) \text {, }
\end{aligned}
$$

and

$$
M(m, 2 r+2, r)=\left(\begin{array}{ccccc}
0 & I^{(r)} & & & \\
& 0 & & & \\
& & \alpha & 1 & \\
& & \alpha & \\
& & & 0^{(m-2 r-2)}
\end{array}\right)
$$

We say that $P$ is a subspace of type $(m, 2 r+\gamma, r, \Gamma)$, if $P G_{2 v+\delta} P^{\mathrm{T}}$ is cogredient to $M(m, 2 r+\gamma, r)$, where $\Gamma=1$ or 0 , if $e_{2 v+1} \in P$ or not, respectively, in case $\delta=\gamma=1$. In particular, subspaces of type $(m, 0,0)$ are called $m$-dimensional totally singular subspaces. The subspaces of type $(m, 2 r+1, r, 1)$ exist if and only if $2 r+1 \leq m \leq v+r+1$. The subspace of type $(m, 2 r+1, r, 1)$, which contains subspaces of type $\left(m_{1}, 2 r+1, r, 1\right)$, exists if and only if $2 r+1 \leq m_{1}<m \leq v+r+1$. Let $N\left(m_{1}, 0,0 ; m, 0,0 ; 2 v+\delta\right)$ denote the number of subspaces of type $\left(m_{1}, 0,0\right)$ contained in a given subspace of type $(m, 0,0)$. From [30],

$$
N\left(m_{1}, 0,0 ; m, 0,0 ; 2 v+\delta\right)=\frac{\prod_{i=m-m_{1}+1}^{m}\left(q^{i}-1\right)}{\prod_{i=1}^{m_{1}}\left(q^{i}-1\right)}
$$


- Suppose $P$ is a subspace of type $(m, r)$ in $2 v$ dimension symplectic space $\mathbb{F}_{q}^{(2 v)}$. A coset of $\mathbb{F}_{q}^{(2 v)}$ relative to a subspace $P$ of type $(m, r)$ is called a $(m, r)$-flat. A flat $F_{1}$ is said to be incident with a flat $F_{2}$, if $F_{1}$ contains or is contained in $F_{2}$. The point set $\mathbb{F}_{q}^{(2 v)}$ with all the flats and the incidence relation among them defined above is said to be the $2 v$-dimensional affine-symplectic space, denoted by $\operatorname{ASG}\left(2 v, \mathbb{F}_{q}\right)$.

The set of matrices of the form

$$
\left(\begin{array}{ll}
T & 0 \\
v & 1
\end{array}\right)
$$

where $T \in S p_{2 v}\left(\mathbb{F}_{q}\right)$ and $v \in \mathbb{F}_{q}^{(2 v)}$, forms a group under matrix multiplication. This group is said to be the affine-symplectic group of $\operatorname{ASG}\left(2 v, \mathbb{F}_{q}\right)$, denoted by $\operatorname{ASp}_{2 v}\left(\mathbb{F}_{q}\right)$. Define the action of $\operatorname{ASp}_{2 v}\left(\mathbb{F}_{q}\right)$ on the $\operatorname{ASG}\left(2 v, \mathbb{F}_{q}\right)$ as follows:

$$
\begin{aligned}
\operatorname{ASG}\left(2 v, \mathbb{F}_{q}\right) \times \operatorname{ASp}_{2 v}\left(\mathbb{F}_{q}\right) & \rightarrow \operatorname{ASG}\left(2 v, \mathbb{F}_{q}\right) \\
\left(x,\left(\begin{array}{ll}
T & 0 \\
v & 1
\end{array}\right)\right) & \mapsto x T+v .
\end{aligned}
$$

Then affine-symplectic group $\operatorname{ASp}_{2 v}\left(\mathbb{F}_{q}\right)$ acts transitive on the set of $(m, r)$-flats in $\operatorname{ASG}\left(2 v, \mathbb{F}_{q}\right)[30]$.

For more information about geometry of classical groups over finite fields, see [30]

\section{The constructions}

\subsection{The constructions based on the vector space}

\subsubsection{Use subspaces of the finite vector space}

Consider the $m$-dimensional space, or simply $m$-space, of $\mathbb{F}_{q}^{(n)}$ where $q$ is a prime or a prime power. Let $\left[\begin{array}{l}m \\ k\end{array}\right]_{q}$ denote the number of $k$-dimensional subspaces, or simply $k$-space. It is well known [29] (p. 291) that the following is true.

\section{Lemma 3.1}

$$
\left[\begin{array}{c}
m \\
k
\end{array}\right]_{q}=\frac{\left(q^{m}-1\right)\left(q^{m-1}-1\right) \cdots\left(q^{m-k+1}-1\right)}{\left(q^{k}-1\right)\left(q^{k-1}-1\right) \cdots(q-1)}
$$

and

$$
\left[\begin{array}{c}
m \\
k
\end{array}\right]_{q}=\left[\begin{array}{c}
m \\
m-k
\end{array}\right]_{q}
$$

Definition 3.2 Fix integers $1 \leq r<k<m$. Let $M(m, k, r)$ be the 01-matrix by taking all $k$-spaces (from an underlying $m$-space) as columns and all $r$-spaces as rows. $M(m, k, r)$ has a 1 in row $i$ and column $j$ if and only if $i$ is contained in $j$. 
$M(m, k, r)$ was first studied by Yakir [32] from a linear algebra point of view and by Ngo and Du [27] from a pooling design point of view. $M(m, k, r)$ is easily checked to be a ranked atomic semilattice, thus the matrix is $r$-disjunct and hence (Huang and Weng [12]) $d^{z}$-disjunct for some $1 \leq d \leq r$ and

$$
z=\left[\begin{array}{l}
k-d \\
r-d
\end{array}\right]_{q}
$$

Note that the construction still requires the row rank $r$ being at least as large as the upper bound $d$ of the number of positive clones. D'yachkov et al. obtained the following theorem.

Theorem 3.3 [2] Suppose $k-r \geq 2$ and set $p:=\frac{q\left(q^{k-1}-1\right)}{q^{k-r}-1}$. Then $M(m, k, r)$ is $d^{z}$-disjunct for $1 \leq d \leq p$ and

$$
z=q^{k-r}\left[\begin{array}{l}
k-1 \\
r-1
\end{array}\right]_{q}-(d-1) q^{k-r-1}\left[\begin{array}{l}
k-2 \\
r-1
\end{array}\right]_{q}
$$

Nan and Guo [25] generalized Ngo and Du's construction [27] and obtained a family of pooling designs

Let $\mathcal{M}(m, n)$ be the set of all $m$-dimensional subspaces of $\mathbb{F}_{q}^{(n)}$.

Definition 3.4 [25] Given integers $1 \leq r, m \leq n-1$ and $\max \{0, r+m-n\} \leq j \leq$ $\min \{r, m\}$. Let $M(r, m ; n)$ be the binary matrix whose rows (resp. columns) are indexed by $\mathcal{M}(r, n)($ resp. $\mathcal{M}(m, n))$. We also order elements of these sets lexicographically. $M(r, m ; n)$ has a 1 in row $i$ and column $l$ if and only if the $i$-th subspace of $\mathcal{M}(r, n)$ intersect the $l$-th subspace of $\mathcal{M}(m, n)$ at $j$-dimensional subspaces of $\mathbb{F}_{q}^{(n)}$.

Then $M(r, m ; n)$ is an $\left[\begin{array}{l}n \\ r\end{array}\right]_{q} \times\left[\begin{array}{l}n \\ m\end{array}\right]_{q}$ matrix, whose constant row (resp. column) weight is $p_{j, j}^{r}(r, m ; n)=q^{(r-j)(m-j)}\left[\begin{array}{l}n-r \\ m-j\end{array}\right]_{q}\left[\begin{array}{l}r \\ j\end{array}\right]_{q}\left(\operatorname{resp} . p_{j, j}^{m}(m, r ; n)=\right.$ $\left.q^{(r-j)(m-j)}\left[\begin{array}{c}n-m \\ r-j\end{array}\right]_{q}\left[\begin{array}{c}m \\ j\end{array}\right]_{q}\right)$.

Theorem 3.5 [25] Let $1 \leq r, m \leq n-1$ and $\max \{0, r+m-n\} \leq j \leq \min \{r, m\}$. If $1 \leq d \leq\left\lfloor p_{j, j}^{m}(m, r ; n) / \alpha\right\rfloor+1$, then $M(r, m ; n)$ is $d^{e}$-disjunct, where $e=$ $p_{j, j}^{m}(m, r ; n)-d \alpha-1, \alpha=\max \left\{p_{j, j}^{l}(m, r ; n) \mid \max \{0,2 m-n\} \leq l \leq m-1\right\}$.

The error-tolerance property of $M(r, m ; n)$ is not well expressed. Guo and Wang [6] constructed a family pooling designs whose error-tolerance property is better than that of [25].

Definition 3.6 [6] For positive integers $1 \leq d<k<n$ and $\max \{0, d+k-n\} \leq i \leq$ $d$, let $M_{q}(i ; d, k, n)$ be the binary matrix by taking all $k$-spaces (from an underlying $n$-space) as columns and all $d$-spaces as rows such that $M_{q}(A, B)=1$ if and only if $\operatorname{dim}(A \cap B)=i$. 
Theorem 3.7 [6] Let $i, d, k, n$ be positive integers with $\lfloor(d+1) / 2\rfloor \leq i \leq d<k$ and $n-k-\bar{s}(k+d-2 i) \geq d-i$. If $k-i \geq 2$ and $1 \leq \bar{s} \leq q\left(q^{k-1}-1\right) /\left(q^{\bar{k}-i}-1\right)$, then the following hold:

(i) $M_{q}(i ; d, k, n)$ is an $\bar{s}^{\bar{e}_{2}}$-disjunct matrix, where

$$
\begin{aligned}
\bar{e}_{2}= & q^{(d-i)(k+\bar{s}(k+d-2 i)-i)}\left[\begin{array}{c}
n-k-\bar{s}(k+d-2 i) \\
d-i
\end{array}\right]_{q} \\
& \times\left(q^{k-i}\left[\begin{array}{c}
k-1 \\
i-1
\end{array}\right]_{q}-(\bar{s}-1) q^{k-i-1}\left[\begin{array}{c}
k-2 \\
i-1
\end{array}\right]_{q}\right)-1
\end{aligned}
$$

(ii) for a given $k$, if $i<d$, then $\lim _{n \rightarrow \infty} \frac{\overline{e_{2}}+1}{\overline{e_{1}+1}}=\infty$. where $\overline{e_{1}}=z-1, z$ is as in Theorem 3.3.

The error-tolerance property of [6] is much better than that of [27] under some conditions.

\subsubsection{Use subspaces which join a fixed subspace being the $\mathbb{F}_{q}^{(n)}$}

In 2015, Liu and Gao [20] constructed a family pooling designs. Denote the set of all $i$-subspaces $U$ of vector space $V$ satisfying $U+W=V$ by $M(i ; n, b)$, where $W$ is a fixed $(n-b)$-subspace of $\mathbb{F}_{q}^{(n)}$.

Definition 3.8 [20] Given integers $1 \leq b<l_{1}<l_{2}<n$. Let $M\left(l_{1}, l_{2} ; n, b\right)$ be the binary matrix whose rows (resp. columns) are indexed by $M\left(l_{1} ; n, b\right)$ (resp. $\left.M\left(l_{2} ; n, b\right)\right)$. We also order elements of these sets lexicographically. $M\left(l_{1}, l_{2} ; n, b\right)$ has a 1 in row $i$ and column $j$ if and only if the $i$ th subspace of $M\left(l_{1} ; n, b\right)$ is a subspace of the $j$ th subspace of $M\left(l_{2} ; n, b\right)$.

Theorem 3.9 [20] Suppose $l_{1}>b, l_{2}-l_{1} \geq 2$, and let $p=\frac{\left(\frac{q^{l_{2}}-q^{b}}{q^{l_{2}-l_{1}}-1}-1\right)\left(q^{l_{2}-b-1}-1\right)}{\left(\frac{q^{l_{2}-1}-q^{b}}{q^{l_{2}-l_{1}-1}-1}-1\right)\left(q^{l_{2}-l_{1}-1}-1\right)}$.

Then $M\left(l_{1}, l_{2} ; n, b\right)$ is $d_{z}$-disjunct for $1 \leq d \leq p q^{b}$, and $z=\left(\frac{q^{l_{2}}-q^{b}}{q^{l_{2}-l_{1}-1}}-\right.$ 1) $q^{b\left(l_{2}-l_{1}-1\right)}\left[\begin{array}{c}l_{2}-b-1 \\ l_{1}-b\end{array}\right]_{q}-(d-1)\left(\frac{q^{l_{2}-1}-q^{b}}{q^{l_{2}-l_{1}-1}-1}-1\right) q^{b\left(l_{2}-l_{1}-2\right)}\left[\begin{array}{c}l_{2}-b-2 \\ l_{1}-b\end{array}\right]_{q}$. Moreover, if $1 \leq d \leq \min \left\{p q^{b}, q\right\}$, then $M\left(l_{1}, l_{2} ; n, b\right)$ is full $d^{z}$-disjunct.

The test efficiency of construction is smaller than that of [2] under some conditions.

\subsection{Use subspaces of singular linear space}

Liu and Gao [19] construct a family of error-correcting pooling designs with the incidence matrix of two types of subspaces of singular linear space over finite fields, and exhibit their disjunct properties. 
Definition 3.10 [19] Given integers $0 \leq k \leq l, 0 \leq m-k \leq n, 0 \leq r \leq m-k-2$, Let $M(r ; m, k ; n+l, n)$ be the binary matrix whose rows (resp. columns) are indexed by $\mathcal{M}(r, 0 ; n+l, n)$ (resp. $\mathcal{M}(m, k ; n+l, n)$. We also order elements of these sets lexicographically. $M(r ; m, k ; n+l, n)$ has a 1 in row $i$ and column $j$ if and only if the $i$-th subspace of $\mathcal{M}(r, 0 ; n+l, n)$ is a subspace of the $j$ th subspace of $\mathcal{M}(m, k ; n+$ $l, n)$.

Theorem 3.11 [19] Given integers $0 \leq k<l, 0 \leq m-k \leq n, 0 \leq r \leq m-k-2$ and let $t=N(r, 0 ; m, k ; n+l, n), u=N(r, 0 ; m-1, k ; n+l, n)), v=N(r, 0 ; m-$ $1, k-1 ; n+l, n), x=N(r, 0 ; m-2, k ; n+l, n), y=N(r, 0 ; m-2, k-1 ; n+l, n)$, $z=N(r, 0 ; m-2, k-2 ; n+l, n)$ and $w=\max \{u-x, u-y, u-z, v-x, v-y, v-z\}$, if $1 \leq d \leq\left\lfloor\frac{t-\max \{u, v\}-1}{w}\right\rfloor+1$ then $M(r ; m, k ; n+l, n)$ is $d^{e}$-disjunct, where $e=$ $t-\max \{u, v\}-(d-1) w-1$. In particular, if $1 \leq d \leq \min \left\{\left\lfloor\frac{t-\max \{u, v\}-1}{w}\right\rfloor+1, q+1\right\}$, then $M(r ; m, k ; n+l, n)$ is fully $d^{e}$-disjunct, where $N\left(m_{1}, k_{1} ; m, k ; n+l, n\right)$ is from (3).

\subsection{The constructions based on the symplectic space}

3.3.1 Use subspaces containing a fixed $\left(m_{0}, 0\right)$-space and contained in its dual space

In 2008, Zhang et al. [33] constructed a family pooling designs based on the symplectic space.

Definition 3.12 [33] Select integers $0 \leq m_{0}<r<m \leq v$. Assume that $P_{0}$ is a fixed $\left(m_{0}, 0\right)$-space of $\mathbf{F}_{q}^{(2 v)}$. Let $M$ be the $(0,1)$-matrix by taking all $(m, 0)$-spaces which are contained in $P_{0}^{\perp}$ and contain $P_{0}$ as columns and all $(r, 0)$-spaces which are contained in $P_{0}^{\perp}$ and contain $P_{0}$ as rows. $M$ has a 1 in row $i$ and column $j$ if and only if $i$ is contained in $j$.

Theorem 3.13 [33] Suppose $m-r \geq 2$ and set $b=\frac{q\left(q^{m-m_{0}-1}-1\right)}{q^{m-r}-1}$. Then $M$ is $d^{z}$-disjunct for $1 \leq d \leq b$ and

$$
z=\left[\begin{array}{c}
m-m_{0} \\
r-m_{0}
\end{array}\right]-d\left[\begin{array}{c}
m-m_{0}-1 \\
r-m_{0}
\end{array}\right]+(d-1)\left[\begin{array}{c}
m-m_{0}-2 \\
r-m_{0}
\end{array}\right]
$$

The test efficiency of construction is smaller than that of [2] under some conditions.

\subsubsection{Use subspaces containing a fixed $\left(d_{0}, r\right)$-space}

In 2010, Li et al. [16] constructed two family pooling designs based on the symplectic space.

Definition 3.14 [16] For $2 r \leq d_{0}<d<k \leq v+r$, assume that $P_{0}$ is a fixed $\left(d_{0}, r\right)$-space of $\mathbb{F}_{q}^{(2 v)}$. Let $M$ be a binary matrix whose columns (rows) indexed by all $(k, r)$-spaces containing $P_{0}\left((d, r)\right.$-spaces containing $\left.P_{0}\right)$ in $\mathbb{F}_{q}^{(2 v)}$ such that $M(A, B)=$ 1 if $A \subseteq B$ and 0 otherwise. This matrix is denoted by $M_{1}(v, d, k)$. 
Theorem 3.15 [16] Suppose $2 r \leq d_{0}<d<k \leq v+r$ and set $b=\frac{q\left(q^{k-d_{0}-1}-1\right)}{q^{k-d}-1}$. Then $M_{1}(\nu, d, k)$ is $s^{e}$-disjunct for $1 \leq d \leq b$ and

$$
\begin{aligned}
e= & q^{k-d} N\left(d-d_{0}-1,0 ; k-d_{0}-1,0 ; 2\left(v+r-d_{0}\right)\right) \\
& -(s-1) q^{k-d-1} N\left(d-d_{0}-1,0 ; k-d_{0}-2,0 ; 2\left(v+r-d_{0}\right)\right),
\end{aligned}
$$

where $N\left(m_{1}, r ; m, r ; 2 v\right)$ is from (4).

The test efficiency of construction is smaller than that of [2] under some conditions.

Definition 3.16 [16] For $2 \leq 2 r \leq d<k \leq v+r$, let $M$ be a binary matrix whose columns (rows) indexed by all subspaces of type $(k, r)((d, r))$ in $\mathbb{F}_{q}^{(2 v)}$ such that $M(A, B)=1$ if $A \subseteq B$ and 0 otherwise. This matrix is denoted by $M_{2}(v, d, k)$.

Theorem 3.17 [16] Suppose $4 \leq 2 r+2 \leq d<k-1 \leq v+r-1$. If $1 \leq s \leq q^{2 r}$, then $M_{2}(v, d, k)$ is $s^{e}$-disjunct, where $e=q^{(k-d-1) d+2 r}$.

The test efficiency of construction is smaller than that of [2] under some conditions.

\subsubsection{Use totally isotropic subspaces and non-totally isotropic subspaces}

In 2010, Guo et al. [8] constructed a family of inclusion matrices associated with subspaces of the symplectic space $\mathbb{F}_{q}^{(2 v)}$.

Definition 3.18 [8] Given integers $1 \leq r \leq m<v$. Let $M(r, 2 m ; 2 v)$ be the binary matrix whose rows (resp. columns) are indexed by $M(r, 0 ; 2 v)(\operatorname{resp} . M(2 m, m ; 2 v))$. We also order elements of these sets lexicographically. $M(r, 2 m ; 2 v)$ has a 1 in row $i$ and column $j$ if and only if the $i$-th subspace of $M(r, 0 ; 2 v)$ is a subspace of the $j$-th subspace of $M(2 m, m ; 2 v)$.

Theorem 3.19 [8] Let $1 \leq r \leq m<v$, and let $\beta=N(r, 0 ; 2 m, m ; 2 v), \gamma=$ $N(r, 0 ; 2 m-1, m-1 ; 2 v), \delta=N(r, 0 ; 2 m-2, m-1 ; 2 v), \xi=N(r, 0 ; 2 m-$ $2, m-2 ; 2 \nu)$ and $\alpha=\max \{\gamma-\delta, \gamma-\xi\}$, where $N\left(m_{1}, r ; m, r ; 2 \nu\right)$ is from (4). Then the following (i)-(iii) hold:

(i) For $m \geq 2$ and $m \geq r+1$, if $1 \leq d \leq\left\lfloor\begin{array}{c}\beta-\gamma-1 \\ \alpha\end{array}\right\rfloor+1$, then $M(r, 2 m ; 2 v)$ is $d^{e}$-disjunct, where $e=\beta-\gamma-(d-1) \alpha-1$. In particular, if $1 \leq d \leq$ $\min \left\{\left\lfloor\begin{array}{c}\beta-\gamma-1 \\ \alpha\end{array}\right\rfloor+1, q+1\right\}$, then $M(r, 2 m ; 2 v)$ is fully $d^{e}$-disjunct.

(ii) For $m \geq 2$ and $m=r$, if $1 \leq d \leq\left\lfloor\begin{array}{c}\beta-1 \\ \gamma\end{array}\right\rfloor$, then $M(r, 2 m ; 2 v)$ is $d^{e}$-disjunct, where $e=\beta-d \gamma-1$. In particular, if $1 \leq d \leq \min \left\{\left\lfloor\begin{array}{c}\beta-1 \\ \gamma\end{array}\right\rfloor, q+1\right\}$, then $M(r, 2 m ; 2 v)$ is fully $d^{e}$-disjunct.

(iii) For $m=1$, if $1 \leq d \leq q$, then $M(1,2 ; 2 v)$ is fully $d^{e}$-disjunct, where $e=q-d$.

The test efficiency of construction is smaller than that of [33] under some conditions. 


\subsubsection{Use totally isotropic subspaces}

In 2014, Guo and Nan [11] generalized [8]. They constructed a new family pooling designs based on the symplectic space.

Definition 3.20 [11] Given integers $1 \leq r \leq\left\lfloor\begin{array}{c}m \\ 2\end{array}\right\rfloor, 2 s \leq m \leq v+s$ and $s+r \leq$ $m<2 v$. Let $M(r, 0 ; m, s ; 2 v)$ be the binary matrix whose rows (resp. columns) are indexed by $M(r, 0 ; 2 v)$ (resp. $M(m, s ; 2 v)) . M(r, 0 ; m, s ; 2 v)$ has a 1 in row $i$ and column $j$, if and only if the $i$ th subspace of type $(r, 0)$ is contained in the $j$ th subspace of type $(m, s)$.

Theorem 3.21 [11] Let $1 \leq r \leq\left\lfloor\begin{array}{c}m \\ 2\end{array}\right\rfloor, s+r \leq m<2 v$, and let $\beta=$ $N(r, 0 ; m, s ; 2 v), \gamma=N(r, 0 ; m-1, s ; 2 \nu), \xi=N(r, 0 ; m-1, s-1 ; 2 \nu), \eta=$ $N(r, 0 ; m-2, s ; 2 \nu), \zeta=N(r, 0 ; m-2, s-1 ; 2 \nu), \delta=N(r, 0 ; m-2, s-2 ; 2 \nu), \alpha=$ $\max \{\gamma-\eta, \gamma-\zeta, \gamma-\delta, \xi-\eta, \xi-\zeta, \xi-\delta\}$, where $N\left(m_{1}, r ; m, r ; 2 \nu\right)$ is from (4). If $d \leq\left\lfloor\frac{\beta-\max \{\gamma, \xi\}-1}{\alpha}\right\rfloor+1$, then $M(r, 0 ; m, s ; 2 v)$ is $d^{e}$-disjunct, where $e=$ $\beta-\max \{\gamma, \xi\}-(d-1) \alpha-1$. In particular, if $1 \leq d \leq \min \left\{\left\lfloor\frac{\beta-\max \{\gamma, \xi\}-1}{\alpha}\right\rfloor+1, q+1\right\}$, then $M(r, 0 ; m, s ; 2 v)$ is fully $d^{e}$-disjunct.

The error-tolerance property of [11] is much better than that of [8], if $v$ is big enough.

\subsection{The construction based on the pseudo-symplectic space}

In 2010, Li et al. [18] constructed two family pooling designs on pseudo-symplectic spaces $\mathbb{F}_{q}^{(2 v+1)}$.

Definition 3.22 [18] For $2 r+1 \leq d_{0}<d<k \leq v+r+1$, assume that $P_{0}$ is a fixed subspace of type $\left(d_{0}, 2 r+1, r, 0\right)$ in $\mathbb{F}_{q}^{(2 v+1)}$. Let $M$ be a binary matrix whose columns (rows) are indexed by all subspaces of type $(k, 2 r+1, r, 0)$ containing $P_{0}$ (subspaces of type $(d, 2 r+1, r, 0)$ containing $\left.P_{0}\right)$ in $\mathbb{F}_{q}^{(2 v+1)}$ such that $M(A, B)=1$ if $A \subseteq B$, and 0 otherwise. This matrix is denoted by $M_{1}(n, d, k)$.

Theorem 3.23 [18] Suppose $2 r+1 \leq d_{0}<d<k \leq v+r+1$ and set $b=$ $\frac{q\left(q^{k-d_{0}-1}-1\right)}{q^{k-d}-1}$. Then $M_{1}(n, d, k)$ is $s^{e}$-disjunct for $1 \leq s \leq b$ and

$$
\begin{aligned}
e= & q^{k-d} N\left(d-d_{0}-1,0,0,0 ; k-d_{0}-1,0,0,0 ; 2\left(v-r+d_{0}\right)\right) \\
& -(s-1) q^{k-d-1} N\left(d-d_{0}-1,0,0,0 ; k-d_{0}-2,0,0,0 ; 2\left(v-r+d_{0}\right)\right),
\end{aligned}
$$

where $N\left(m_{1}, 0,0,0 ; m, 0,0,0 ; 2 v+\delta\right)$ is from (5).

Definition 3.24 For $3 \leq 2 r+1 \leq d<k \leq v+r+1$, let $M$ be a binary matrix whose columns (rows) are indexed by all subspaces of type $(k, 2 r+1, r, 1)((d, 2 r+1, r, 1))$ in $\mathbb{F}_{q}^{(2 v+1)}$ such that $M(A, B)=1$ if $A \subseteq B$, and 0 otherwise. This matrix is denoted by $M_{2}(n, d, k)$. 
Theorem 3.25 [18] Suppose $3 \leq 2 r+1 \leq d-1<k-2 \leq v+r+1$. If $1 \leq s \leq q^{2 r}$, then $M_{2}(n, d, k)$ is $s^{e}$-disjunct, where $e=q^{(k-d-1)(d-1)+2} r$.

The test efficiency of construction is smaller than that of [2] under some conditions.

\subsection{The constructions based on the unitary space}

3.5.1 Use subspaces containing a fixed $\left(m_{0}, 0\right)$-space and contained in its dual space

In 2009, Zhang et al. [34] constructed a family pooling designs based on the unitary space.

Definition 3.26 [34] Select integers $0 \leq m_{0}<r<m \leq v$. Assume $P_{0}$ is a fixed $\left(m_{0}, 0\right)$-space of $\mathbb{F}_{q^{2}}^{(n)}$. Let $M$ be the $(0,1)$-matrix by taking all $(m, 0)$-spaces which are contained in $P_{0}^{\perp}$ and contain $P_{0}$ as columns and all $(r, 0)$-spaces which are contained in $P_{0}^{\perp}$ and contain $P_{0}$ as rows. $M$ has a 1 in row $i$ and column $j$ if and only if $i$ is contained in $j$.

Theorem 3.27 [34] Suppose $m-r \geq 2$ and set $b=\frac{\left(q^{2\left(m-m_{0}-1\right)}-1\right)\left(q^{2\left(m-m_{0}\right)}-q^{2(m-r-2)}\right)}{\left(q^{2(m-r-2)}-1\right)\left(q^{2\left(m-m_{0}-1\right)}-1\right)-1}$. Then $M$ is $d^{z}$-disjunct for $1 \leq d \leq b$ and

$$
z=\left[\begin{array}{c}
m-m_{0} \\
r-m_{0}
\end{array}\right]_{q^{2}}\left[\begin{array}{c}
m-m_{0}-1 \\
r-m_{0}
\end{array}\right]_{q^{2}}+(d-1)\left[\begin{array}{c}
m-m_{0}-2 \\
r-m_{0}
\end{array}\right]_{q^{2}} .
$$

The test efficiency of construction is smaller than that of [2] under some conditions.

\subsubsection{Use subspaces containing a fixed $\left(d_{0}, r\right)$-space}

In 2011, Gao et al. [10] presented two new constructions based on the unitary space.

Definition 3.28 [10] For $2 r-2 d_{0}<2 d<2 k \leq n+r$, assume that $P_{0}$ is a fixed subspace of type $\left(d_{0}, r\right)$ in $\mathbb{F}_{q^{2}}^{(n)}$. Let $M$ be a binary matrix whose columns (rows) are indexed by all subspaces of type $(k, r)$ containing $P_{0}$ (subspaces of type $(d, r)$ containing $\left.P_{0}\right)$ in $\mathbb{F}_{q^{2}}^{(n)}$ such that $M(A, B)=1$ if $A \subseteq B$ and 0 otherwise. This matrix is denoted by $M_{1}(n, d, k)$.

Theorem 3.29 [10] Suppose $2 r \leq 2 d_{0}<2 d<2 k \leq n+r, r=2 s+\delta_{1}$, where $\delta_{1}=0,1$ and set $b=\frac{q^{2}\left(q^{2\left(k-d_{0}-1\right)}-1\right)}{q^{2(k-d)}-1}$. Then $M_{1}(n, d, k)$ is $l^{e}$-disjunct for $1 \leq l \leq b$ and

$$
\begin{aligned}
e= & q^{2(k-d)} N\left(d-d_{0}-1,0 ; k-d_{0}-1,0 ; 2\left(v+s-d_{0}\right)\right) \\
& -(l-1) q^{2(k-d-1)} N\left(d-d_{0}-1,0 ; k-d_{0}-2,0 ; 2\left(v+s-d_{0}\right)\right),
\end{aligned}
$$

where $N\left(m_{1}, r ; m, r ; n\right)$ is from (6). 
Definition 3.30 [10] For $2 \leq 2 r \leq 2 d<2 k \leq n+r$, let $M$ be a binary matrix whose columns (rows) are indexed by all subspaces of type $(k, r)((d, r))$ in $\mathbb{F}_{q^{2}}^{(n)}$ such that $M(A, B)=1$ if $A \subseteq B$ and 0 otherwise. This matrix is denoted by $M_{2}(n, d, k)$.

Theorem 3.31 [10] Suppose $0 \leq 2 r-4 \leq 2 d<2 k-2 \leq n+r-2$. If $1 \leq s \leq q^{2 r}$, then $M_{2}(n, d, k)$ is $s^{e}$-disjunct, where $e=q^{2(k-d-1) d+2 r}$.

The test efficiency of construction is smaller than that of [2] under some conditions.

\subsubsection{Use totally isotropic subspaces}

In 2010, Guo [4] constructed a family of inclusion matrices associated with subspaces of $\mathbb{F}_{q^{2}}^{(2 v+\delta)}$, and exhibited its disjunct property.

Definition 3.32 [4] In the unitary space $\mathbb{F}_{q^{2}}^{(2 v+\delta)}$, given integers $1 \leq r \leq\left\lfloor\frac{m}{2}\right\rfloor$ and $m<2 v+\delta$. Let $M(r, m ; 2 v+\delta)$ be the binary matrix whose rows (resp. columns) are indexed by $\mathcal{M}(r, 0 ; 2 v+\delta)$ (resp. $\mathcal{M}(m, m ; 2 v+\delta)$ ). We also order elements of these sets lexicographically. $M(r, m ; 2 v+\delta)$ has a 1 in row $i$ and column $j$ if and only if the $i$-th subspace of $\mathcal{M}(r, 0 ; 2 v+\delta)$ is a subspace of the $j$-th subspace of $\mathcal{M}(m, m ; 2 v+\delta)$.

Theorem 3.33 [4] Let $1 \leq r \leq\left\lfloor\frac{m}{2}\right\rfloor$ and $m<2 v+\delta$, and let $\beta=N(r, 0 ; m, m ; 2 v+$ $\delta), \gamma=N(r, 0 ; m-1, m-1 ; 2 v+\delta), \xi=N(r, 0 ; m-1, m-2 ; 2 v+\delta), \eta=$ $N(r, 0 ; m-2, m-2 ; 2 v+\delta), \zeta=N(r, 0 ; m-2, m-3 ; 2 v+\delta), \rho=N(r, 0 ; m-$ $2, m-4 ; 2 v+\delta), \alpha=\max \{\gamma-\eta, \gamma-\zeta, \gamma-\rho, \xi-\eta, \xi-\zeta, \xi-\rho\}$, where $N\left(m_{1}, r ; m, r ; n\right)$ is from (6). Then the following (i)-(iii) hold:

(i) For $m \geq 4$. If $1 \leq d \leq\left\lfloor\frac{\beta-\max \{\gamma, \xi\}-1}{\alpha}\right\rfloor+1$, then $M(r, m ; 2 v+\delta)$ is $d^{e}$ disjunct, where $e=\beta-\max \{\gamma, \xi\}-(d-1) \alpha-1$. In particular, if $1 \leq$ $d \leq \min \left\{\left\lfloor\frac{\beta-\max \{\gamma, \xi\}-1}{\alpha}\right\rfloor+1, q^{2}+1\right\}$, then exist $d+1$ distinct columns of $M(r, m ; 2 v+\delta)$, i.e., $d+1$ distinct $m$-dimensional non-isotropic subspaces of $\mathbb{F}_{q^{2}}^{(2 v+\delta)}$, such that the $d+1$ subspaces contain same $(m-2)$-dimensional subspace $P$ and the number of $r$-dimensional totally isotropic subspaces contained in $P$ is equal to $\min \{\eta, \zeta, \rho\}$.

(ii) For $m=3$. If $1 \leq d \leq q^{2}-q$, then $M(r, m ; 2 v+\delta)$ is fully $d^{e}$-disjunct, where $e=q^{3}-d(q+1)$.

(iii) For $m=2$. If $1 \leq d \leq q$, then $M(1,2 ; 2 v+\delta)$ is fully $d^{e}$-disjunct, where $e=q-d$.

The test efficiency of construction is smaller than that of [2] under some conditions.

\subsection{The constructions based on the orthogonal space}

\subsubsection{Use subspaces containing a fixed $\left(m_{0}, 0,0\right)$-space and contained in its dual space}

In 2009, Zhang et al. [35] constructed a family pooling designs. 
Definition 3.34 [35] Select integers $0 \leq m_{0}<r<m \leq v$. Assume $P_{0}$ is a fixed $\left(m_{0}, 0,0\right)$-subspace of $\mathbb{F}_{q}^{(2 v+\delta)}$. Let $M$ be the $(0,1)$-matrix, where the columns (rows) are labeled by $(m, 0,0)$-subspaces $((r, 0,0)$-subspaces $)$, which are contained in $P_{0}^{\perp}$ and contain $P_{0} \cdot m_{i j}=1$ if and only if the label of row $i$ is contained in the label of column $j$.

Theorem 3.35 [35] Suppose $m-r \geq 2$ and set $b_{1}=\frac{q\left(q^{m-m_{0}-1}-1\right)}{q^{m-r}-1}$. Then $M$ is $d^{z}$-disjunct when $1 \leq d \leq b_{1}$, where

$$
\begin{aligned}
z= & N\left(r-m_{0}, 0,0 ; m-m_{0}, 0,0 ; 2\left(v-m_{0}\right)+\delta, \Delta\right) \\
& -d N\left(r-m_{0}, 0,0 ; m-m_{0}-1,0,0 ; 2\left(v-m_{0}\right)+\delta, \Delta\right) \\
& +(d-1) N\left(r-m_{0}, 0,0 ; m-m_{0}-2,0,0 ; 2\left(v-m_{0}\right)+\delta, \Delta\right)
\end{aligned}
$$

where $N\left(m_{1}, 0,0 ; m, 0,0 ; 2 v+\delta, \Delta\right)$ is from (7).

The test efficiency of construction is smaller than that of [2] under some conditions.

\subsubsection{Use subspaces containing a fixed $\left(d_{0}, 2 r+1, r, 1\right)$-space}

In 2010, Li et al. [17] constructed two family pooling designs.

Definition 3.36 [17] For $2 r+1 \leq d_{0}<d<k \leq v+r+1$, assume that $P_{0}$ is a fixed subspace of type $\left(d_{0}, 2 r+1, r, 1\right)$ in $\mathbb{F}_{q}^{(2 v+1)}$. Let $M$ be a binary matrix whose columns (rows) are indexed by all subspaces of type $(k, 2 r+1, r, 1)$ containing $P_{0}$ ( all subspaces of type $(d, 2 r+1, r, 1)$ containing $\left.P_{0}\right)$ in $\mathbb{F}_{q}^{(2 v+1)}$ such that $M(A, B)=1$ if $A \subseteq B$ and 0 otherwise. This matrix is denoted by $M_{1}(v, d, k)$.

Theorem 3.37 [17] Suppose $2 r+1 \leq d_{0}<d<k \leq v+r+1$ and set $b=$ $\frac{q\left(q^{k-d_{0}-1}-1\right)}{q^{k-d}-1}$. Then $M_{1}(v, d, k)$ is $s^{e}$-disjunct for $1 \leq s \leq b$ and

$$
\begin{aligned}
e= & q^{k-d} N\left(d-d_{0}-1,0,0 ; k-d_{0}-1,0,0 ; 2\left(v+r+1-d_{0}\right)\right) \\
& -(s-1) q^{k-d-1} N\left(d-d_{0}-1,0,0 ; k-d_{0}-2,0,0 ; 2\left(v+r+1-d_{0}\right)\right),
\end{aligned}
$$

where $N\left(m_{1}, 0,0 ; m, 0,0 ; 2 v\right)$ is from (8).

The test efficiency of construction is smaller than that of [2] under some conditions.

Definition 3.38 For $3 \leq 2 r+1 \leq d<k \leq v+r+1$, let $M$ be a binary matrix whose columns (rows) are indexed by all subspaces of type $(k, 2 r+1, r, 1)$ (all subspaces of type $(d, 2 r+1, r, 1))$ in $\mathbb{F}_{q}^{(2 v+1)}$ such that $M(A, B)=1$ if $A \subseteq B$ and 0 otherwise. This matrix is denoted by $M_{2}(v, d, k)$.

Theorem 3.39 [17] Suppose $3 \leq 2 r+1 \leq d-1<k-2 \leq v+r-1$. If $1 \leq s \leq q^{2 r}$, then $M_{2}(v, d, k)$ is $s^{e}$-disjunct, where $e=q^{(k-d-1)(d-1)+2} r$.

The test efficiency of construction is smaller than that of [2] under some conditions. 


\subsection{The constructions based on the affine-symplectic space}

In 2011, Gao et al. [9] constructed two family pooling designs based on the affinesymplectic space.

Definition 3.40 [9] For $2 r-d_{0}<d<k \leq v+r$, assume that $y_{0}+P_{0}$ is a fixed $\left(d_{0}, r\right)$-flat of $\operatorname{ASG}\left(2 v, \mathbb{F}_{q}\right)$. Let $M$ be a binary matrix whose columns (rows) indexed by all $(k, r)$-flats containing $y_{0}+P_{0}\left((d, r)\right.$-flats containing $\left.y_{0}+P_{0}\right)$ in $\operatorname{ASG}\left(2 v, \mathbb{F}_{q}\right)$ ) such that $M(x+A, y+B)=1$ if $x+A \subseteq y+B$ and 0 otherwise. This matrix is denoted by $M_{1}(v, d, k)$.

Theorem 3.41 [9] Suppose $2 r \leq d_{0}<d<k \leq v+r$ and set $b=\frac{q\left(q^{k-d_{0}-1}-1\right)}{q^{k-d}-1}$. Then $M_{1}(v, d, k)$ is $s^{e}$-disjunct for $1 \leq s \leq b$ and

$$
\begin{aligned}
e= & q^{k-d} N\left(d-d_{0}-1,0 ; k-d_{0}-1,0 ; 2\left(v+r-d_{0}\right)\right) \\
& -(s-1) q^{k-d-1} N\left(d-d_{0}-1,0 ; k-d_{0}-2,0 ; 2\left(v+r-d_{0}\right)\right),
\end{aligned}
$$

where $N\left(m_{1}, r ; m, r ; 2 v\right)$ is from (4).

The test efficiency of construction is smaller than that of [2] under some conditions.

Definition 3.42 For $2-2 r-d<k \leq v+r$, let $M$ be a binary matrix whose columns (rows) indexed by all $(k, r)$-flats $\left((d, r)\right.$-flats) in $\operatorname{ASG}\left(2 v, \mathbb{F}_{q}\right)$ such that $M(x+A, y+B)=1$ if $x+A \subseteq y+B$ and 0 otherwise. This matrix is denoted by $M_{2}(v, d, k)$.

Theorem 3.43 [9] Suppose $4 \leq 2 r+2 \leq d<k-1 \leq v+r-1$. If $1 \leq s \leq q^{2 r+1}$, then $M_{2}(v, d, k)$ is $s^{e}$-disjunct, where $e=q^{(k-d-1)(d+1)+2 r+1}$.

The test efficiency of construction is smaller than that of [2] under some conditions.

\section{Conclusion}

The algebraic construction is motivated from containment design which was initiated by Macula [21,22]. Ngo and Du [26,27] generalized containment design to a more general setting. Park et al. [28] employed simplicial complex to perform the idea of containment design. Huang and Weng [12] gave a very general theorem for containment design and suggested to use linear spaces as tools, which initiated the algebraic construction. Note that geometric lattices are among pooling spaces. In [13] the authors gave some examples of pooling spaces from geometry of classical groups over finite fields. So, in this sense the construction based on geometry of classical groups over finite fields was initialed by [13]. This construction opened a new door for studying pooling designs. Current results in literature (Dyachkov et al. [2]; Lang et al. [15]) have showed that many spaces can be involved in algebraic construction. Therefore, the idea is powerful. It may be possible to extend to pooling design of other types with other applications (Lang et al. [15]; Macula et al. [24]). 
Acknowledgements Supported by Natural Science Foundation of China (11471097), the NSF of Hebei Province (No. A2017403010) and the Key Foundation of Hebei Education Department (ZD2017064).

\section{References}

1. Du, D., Hwang, F.K.: Combinatorial Group Testing and Its Applications, 2nd edn. World Scientific, Singapore (2000)

2. D'yachkov, A.G., Hwang, F.K., Macula, A.J., Vilenkin, P.A., Weng, C.: A construction of pooling designs with some happy surprises. J. Comput. Biol. 12, 1129-1136 (2005)

3. Erdös, P., Frankl, P., Füredi, D.: Families of finite sets in which no set is covered by the union of $r$ others. Israel J. Math. 51, 79-89 (1985)

4. Guo, J.: Pooling designs associated with unitary space and ratio efficiency comparison. J. Comb. Optim. 19, 492-500 (2010)

5. Guo, J., Wang, K.S.: A construction of pooling designs with surprisingly high degree of error correction. J. Combin. Theory Ser. A 118, 2056-2058 (2011)

6. Guo, J., Wang, K.S.: Pooling designs with surprisingly high degree of error correction in a finite vector space. Discrete Appl. Math. 160, 2172-2176 (2012)

7. Guo, J., Wang, K.S., Weng, C.W.: Pooling semilattices and non-adaptive pooling designs. Discrete Math. 320, 64-72 (2014)

8. Guo, J., Wang, Y.X., Gao, S.G., Yu, J.C., Wu, W.L.: Constructing error-correcting pooling designs with symplectic space. J. Comb. Optim. 20, 413-421 (2010)

9. Gao, S.G., Li, Z.T., Du, H.J., Shi, Y., Wu, W.L.: Approaching pooling design with smaller efficient ratio. J. Global Optim. 49, 125-135 (2011)

10. Gao, S.G., Li, Z.T., Yu, J.C., Gao, X.F., Wu, W.L.: DNA library screening, pooling design and unitary spaces. Theor. Comput. Sci. 412, 217-224 (2011)

11. Guo, H.X., Nan, J.Z.: Construction of error-tolerance pooling designs in symplectic spaces. J. Global Optim. 58, 405-410 (2014)

12. Huang, T.Y., Weng, C.W.: Pooling spaces and non-adaptive pooling designs. Discrete Math. 282, 163-169 (2004)

13. Huang, T.Y., Wang, K.S., Weng, C.W.: Pooling spaces associated with finite geometry. European J. Combin. 29, 1483-1491 (2008)

14. Kautz, W.H., Singleton, R.C.: Nonadaptive binary superimposed codes. IEEE Trans. Inform. Theory 10, 363-377 (1964)

15. Lang, W., Wang, Y., Yu, J., Gao, S.G., Wu, W.L.: Error-tolerant trivial two-stage group testing for complexes using almost separable almost disjunct martrices. Discrete Math. Algorithms Appl. 1, 235251 (2009)

16. Li, Z.T., Huang, T.Y., Gao, S.G.: Two error-correcting pooling designs from symplectic spaces over a finite field. Linear Algebra Appl. 433, 1138-1147 (2010)

17. Li, Z.T., Gao, S.G., Du, H.J., Zou, F., Wu, W.L.: Two constructions of new error-correcting pooling design from orthogonal spaces over finite field of characteristic 2. J. Comb. Optim. 20, 325-334 (2010)

18. Li, Z.T., Gao, S.G., Du, H.J., Zou, F., Wu, W.L.: Efficient error-correcting pooling designs constructed from pseudo-symplictic spaces over a finite field. J. Comput. Biol. 17, 1413-1423 (2010)

19. Liu, X.M., Gao, Y.: Constructing error-correcting pooling designs with singular linear space. J. Combin. Math. Combin. Comput. 87, 267-274 (2013)

20. Liu, X.M., Gao, X.: New error-correcting pooling designs with vector spaces over finite field. Discrete Math. 338, 857-862 (2015)

21. Macula, A.J.: A simple construction of $d$-disjunct matrices with certain constant weights. Discrete Math. 162, 311-312 (1996)

22. Macula, A.J.: Error-correcting nonadaptive group testing with $d^{e}$-disjunct matrices. Discrete Appl. Math. 80, 217-222 (1997)

23. Macula, A.J.: Probabilistic nonadaptive and two-stage group testing with relatively small pools and DNA library screening. J. Comb. Optim. 2, 385-397 (1998)

24. Macula, A.J., Gal, S., Andam, C., Bishop, M.A., Renz, T.E.: PCR nonadaptive group testing of DNA libraries for biomolecular computing and taggant applications. Discrete Math. Algorithms Appl. 1, 59-69 (2009) 
25. Nan, J.Z., Guo, J.: New error-correcting pooling designs associated with finite vector spaces. J. Comb. Optim. 20, 96-100 (2010)

26. Ngo, H., Du, D.: A survey on combinatorial group testing algorithms with applications to DNA library screening. Discrete Math. Theor. Comput. Sci. 55, 171-182 (2000)

27. Ngo, H., Du, D.: New constructions of non-adaptive and error-tolerance pooling designs. Discrete Math. 243, 161-170 (2002)

28. Park, H., Wu, W., Liu, Z., Wu, X., Zhao, H.G.: DNA screening, pooling design and simplicial complex. J. Comb. Optim. 7(4), 389-394 (2003)

29. van Lint, J.H., Wilson, R.M.: A Course in Combinatorics. Cambridge, Victoria (1992)

30. Wan, Z.X.: Geometry of Classical Groups Over Finite Fields, 2nd edn. Science Press, Beijing (2002)

31. Wang, K.S., Guo, J., Li, F.: Singular linear space and its applications. Finite Fields Appl. 17, 395-406 (2011)

32. Yakir, A.: Inclusion matrix of $k$ vs 1 affine subspaces and a permutation module of the general affine group. J. Combin. Theory Ser. A 63, 301-317 (1993)

33. Zhang, G.S., Li, B.L., Sun, X.L., Li, F.: A construction of $d^{z}$-disjunct matrices in a dual space of symplectic space. Discrete Appl. Math. 156, 2400-2406 (2008)

34. Zhang, G.S., Sun, X.L., Li, B.L.: Error-correcting pooling designs associated with the dual space of unitary space and ratio efficiency comparison. J Comb. Optim. 18, 51-63 (2009)

35. Zhang, G.S., Yang, Y.Q., Zhao, X.H.: A construction of $d^{z}$-disjunct matrices by orthogonal space and discussion on their design parameters. Discrete Math. 309, 6026-6034 (2009) 